\title{
A FEASIBILITY STUDY ON USE OF GENERIC MOBILE LASER SCANNING SYSTEM FOR DETECTING ASPHALT PAVEMENT CRACKS
}

\author{
Xinqu Chen ${ }^{\mathrm{a}}$, Jonathan $\mathrm{Li}^{\mathrm{a}}$ \\ ${ }^{a}$ Mobile Sensing and Geodata Science Lab, Department of Geography and Environmental Management, University of Waterloo, 200 \\ University Avenue West, Waterloo, Ontario N2L 3G1, Canada - (x383chen, junli)@uwaterloo.ca
}

\section{Commission I, ICWG I/Va}

KEY WORDS: Mobile Laser Scanning, Point Cloud, Pavement Crack, Automated Detection, Urban Road

\begin{abstract}
:
This study aims to automatically detect pavement cracks on urban roads by employing the 3D point clouds acquired by a mobile laser scanning (MLS) system. Our method consists of four steps: ground point filtering, high-pass convolution, matched filtering, and noise removal. First, a voxel-based upward growing method is applied to construct Digital Terrain Model (DTM) of the road surface. Then, a high-pass filter convolutes the DTM to detect local elevation changes that may embed cracking information. Next, a two-step matched filter is applied to extract crack features. Lastly, a noise removal process is conducted to refine the results. Instead of using MLS intensity, this study takes advantages of the MLS elevation information to perform automated crack detection from large-volume, mixed-density, unstructured MLS point clouds. Four types of cracks including longitudinal, transvers, random, and alligator cracks are detected. Our results demonstrated that the proposed method works well with the RIEGL VMX-450 point clouds and can detect cracks in moderate-to-severe severity $(13-25 \mathrm{~mm}$ ) within a $200 \mathrm{~m}$ by $30 \mathrm{~m}$ urban road segment located in Kingston, Ontario, at one time. Due to the resolution capability, small cracks with slight severity remain unclear in the MLS point cloud.
\end{abstract}

\section{INTRODUCTION}

In Canada, trucks transport $90 \%$ of all exports to the United States. About 11 million trucks cross the Canada-United States border every year (Smith et al., 2001). In terms of traffic loads, these trucks have a significant impact on pavement performance and can result in pavement cracking and deformation. These heavy loads combined with warm summers and cold winters, result in very demanding requirements for frequent pavement inspection of Canada's aging transportation infrastructure, particularly in those border areas. According to Public Works Canada, over 10 billion dollars are spent on pavements annually in Canada. Frequent inspection and proper maintenance are highly recommended to ensure driver's safety and extend pavement's life. Pavement condition is traditionally assessed by in-situ measurements which rely heavily on manual data collection followed by visual evaluation based on given pavement condition rating manuals. Such pavement condition survey is often time-consuming, subjective, dangerous, and expensive that demands heavily for professional evaluators. Current practices have widely adopted image-based technology, which captures photo logging by high resolution vehicle-mounted cameras, to achieve automated data collection. However, the quality of digital images varies with lighting conditions and existence of shadows which requires high-level image processing methods to overcome.

With the blooming of vehicle-borne mobile laser scanning (MLS) techniques in recent years, MLS applications have developed rapidly in every part of urban road management. Especially, MLS data provides valuable 3D road information to transportation agencies. By extracting road objects from MLS point clouds along the vehicle trajectory, it has great aid in monitoring pavement condition, traffic safety and road sign system (Yu et al., 2016a; 2016b). In our previous publications, we have presented several methods for extraction of various road surface's objects such as road boundaries (Wang et al., 2015), road markings (Yang et al., 2012; Guan et al., 2014; Guan et al., 2015; Yu et al., 2015c), pavement cracks (Guan et al., 2015), road manholes (Yu et al., 2014; Yu et al., 2015a), traffic signs (Wen et al., 2016; Yu et al., 2016b), street light poles (Yu et al., 2015b; Wu et al., 2016), and parking cars (Yu et al., 2016c). Traditionally, digital images were the preferred source of road crack surveying which can derive road cracks in millimetre width. (e.g., Xu and Huang, 2003). However, image qualities often depend on weather, traffic, and image photogrammetry techniques (Nguyen et al., 2011). Pavement crack detection using MLS point clouds becomes a new research topic recently. The idea was basically to make the 3D information of MLS data aid in pavement distress analysis. However, the large volume, mixed-density and irregular distribution of 3D MLS points make road surface's object extraction very challenging and difficult. Our efforts have been placed on development of novel algorithms and software tools for accurate and robust object extraction. To achieve this goal, current MLS road crack extraction methods rely on converting 3D pavement information into 2D image-based data and crack delineation is performed using image processing approaches. Previous studies on pavement cracks detection can achieve precision in centimetre using direct detection from 3D point clouds. Guan et al. (2014) proposed an iterative tensor voting algorithm to detect road cracks from geo-referenced images. The iterative tensor voting based crack detection achieved completeness as $96 \%$ and correctness as $85 \%$. It could well detect road cracks with width larger than $2 \mathrm{~cm}$. Tsai and $\mathrm{Li}$ (2012) implemented a dynamic-optimization-based crack segmentation method on high-resolution 3D continuous transverse pavement profiles. The continuous transverse pavement profiles acquired from a MLS system could aid in detecting road cracks with widths greater than $2 \mathrm{~mm}$. It enabled crack detection under low intensity contrast and lighting conditions with above $95 \%$ score of precision. The installation of both downward camera and the 360 degrees laser scanning could be available to traffic safety maintenance.

Currently, generic MLS system such as RIEGL VMX-450 has demonstrated its potential in pavement inspection. The system is capable of collecting high-density and high-accuracy $3 \mathrm{D}$ point clouds covering the road corridors at a normal driving speed. Generic MLS systems have several advantages over the 
Automatic Road Analyzer (ARAN), a specifically designed mobile pavement condition mapping system, in terms of scanning range and application diversity. Still, it is needed to address that whether generic MLS systems could meet the Ministry of Transportation Ontario (MTO)'s requirements for pavement condition mapping or not. This study aims to automatically detect pavement cracks by employing the 3D point clouds covering a typical urban street in Kingston, Ontario, acquired by a RIEGL VMX-450 system.

\section{STUDY AREA AND DATA ACQUISITION}

The study area consists of one part of the Front Road in the City of Kingston, Ontario, Canada $\left(76^{\circ} 33^{\prime} 22.57^{\prime \prime} \mathrm{W}, 44^{\circ} 13^{\prime} 7.40^{\prime \prime N}\right.$, and see Figure 1). The road segment is approximately $200 \mathrm{~m}$ long and $12 \mathrm{~m}$, containing four lanes (two lanes at each direction) (see Figure 2). The MLS point clouds covered the study area were acquired by a RIEGL VMX-450 system on August 29, 2013. It collected a total of 25,544,329 points and captured images from four side views along the road survey. The point spacing is $3.4 \mathrm{~cm} /$ point

Pavement cracks are presented in the study area including longitudinal, transverse, longitudinal, transvers, and random cracks (see Figure 3).

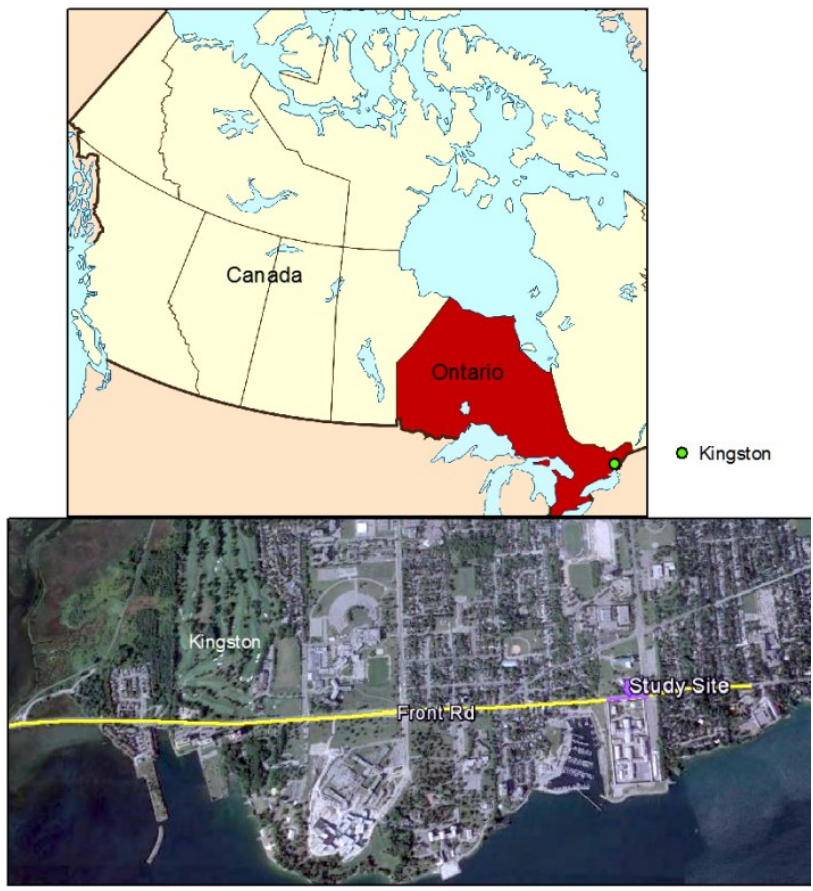

Figure 1. Location of the study area, Kingston, Ontario, Canada

\section{PAVEMENT CRACK DETECTION}

The proposed method mainly consists of four steps: ground point filtering, DTM high-pass convolution, matched filtering, and post noise removal. First, a voxel-based upward growing method (Yu et al., 2015b) is applied to detect the ground points (see Figure 4). The point cloud will be first segmented into a series of blocks according to a user-defined width as shown in Figure 4(a). The voxel-based upward growing algorithm is performed on each segment. Within each block, points are spatially grouped into voxels (see Figure 4(b)). A voxel is defined as a ground voxel is it has nine neighbours. For instance, as shown in Figure 4(c), $\mathrm{V}_{1}$ has nine neighbours: $\mathrm{L}_{1}, \mathrm{~L}_{2} \ldots, \mathrm{L}$. The ground point filtering is an upward growing process which will stop if either no more voxels will have nine neighbours or

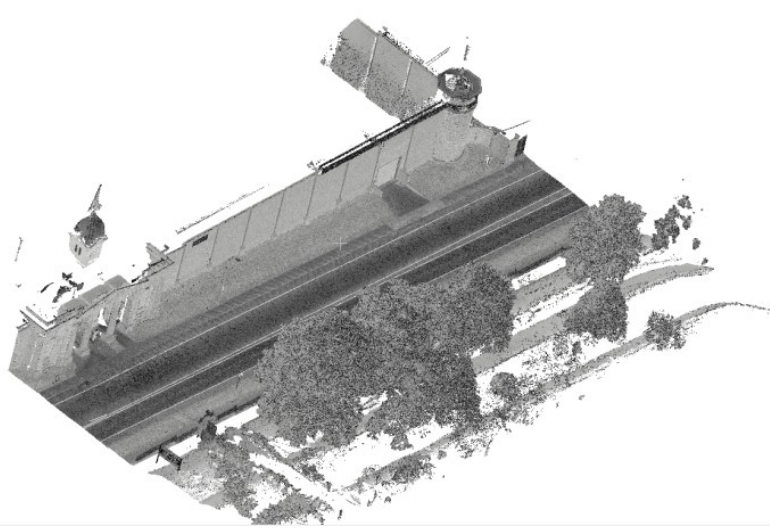

Figure 2. Point clouds acquired by RIEGL VMX-450.

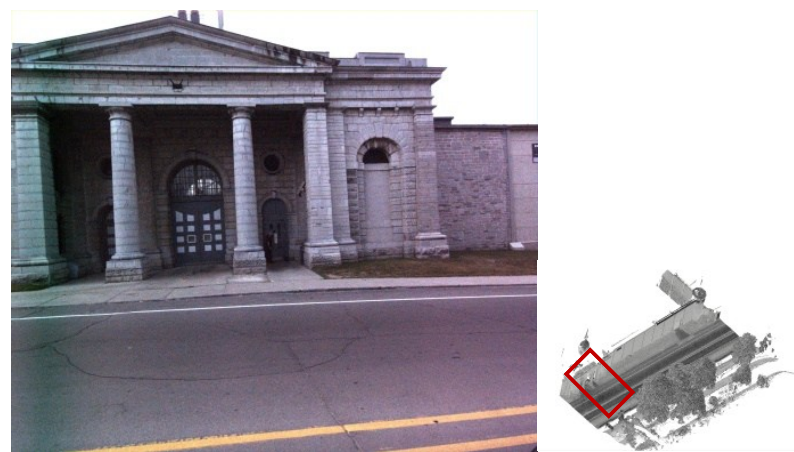

Figure 3. A pavement image taken by the digital camera.

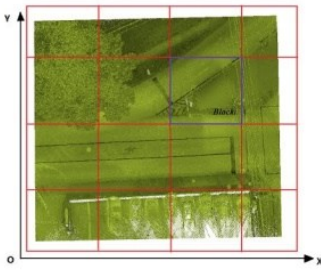

(a)

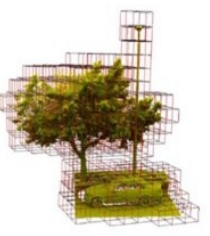

(b)

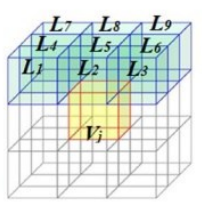

(c)
Figure 4. Principle of the voxel-based upward-growing algorithm (Adapted from Yu et al., 2015b): (a) segmented raw point cloud, (b) octree spatial index in a local block, and (c) voxel-based upward-growing pattern

the elevation between the top and the bottom voxel exceeds the user-defined threshold. In the next step, a DTM raster data was generated by rasterizing all the ground points into $4 \mathrm{~cm}$ grids by assigning the minimum height within the grid as the pixel value. For the grids which had no point filled in, the grid values were interpolated linearly by searching the neighbours. In this way, the points that represent the cracks could be largely reserved. Next, a $5 \times 5$ high-pass filter convolutes the DTM to detect local elevation change. Since the pavement surface with no cracks will have even roughness and complete coverage, the use of a high pass filter can amplify the magnitude of a small elevation jump or detect the surface that is rougher than the rests. These features may embed cracking information. Hence, the ideal high pass filter is applied to the DTM (see Figure 5) and the absolute value of the convolution result is taken. The ideal high-pass filter assigns the difference between the centre grid and the mean of its 24 neighbours. The window size is determined by 


\begin{tabular}{|r|r|r|r|r|}
\hline-1 & -1 & -1 & -1 & -1 \\
\hline-1 & -1 & -1 & -1 & -1 \\
\hline-1 & -1 & 24 & -1 & -1 \\
\hline-1 & -1 & -1 & -1 & -1 \\
\hline-1 & -1 & -1 & -1 & -1 \\
\hline
\end{tabular}

Figure 5 . The $5 \times 5$ ideal high-pass kernel.

taking both the cell size and the common crack size into consideration and is tested empirically. An elevation jump could occur due to the presence of road curb, grass at the sidewalk, or the cracking. A user-defined threshold is used here to retain those small elevation changes within a few centimetres and eliminate those changes at the roadsides. Figure 6 shows an example of the derived DTM high-pass convolution result.

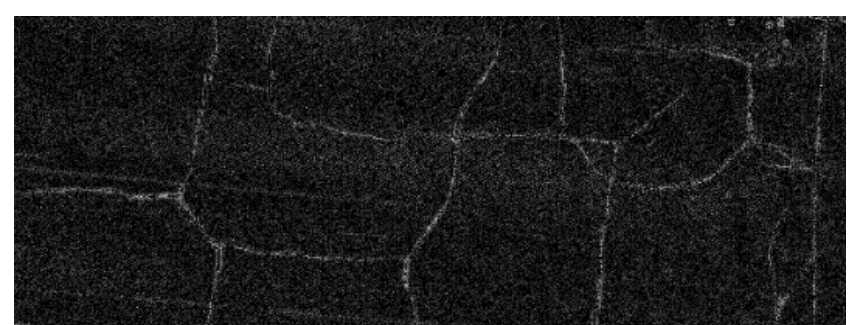

Figure 6. Illustration of the derived DTM high-pass convolution result.

To delineate pavement cracks, a two-step matched filter is applied to extract crack features. The matched filter was first proposed by Chaudhuri et al. (1970) and applied on retinal images to detect vessels. The algorithm assumes that vessels can be described by a Gaussian function as

$$
\mathrm{f}(\mathrm{x}, \mathrm{y})=A\left[1-k \exp \left(\frac{-d^{2}}{2 \sigma^{2}}\right)\right]
$$

where $f(x, y)=$ grayscale elevation at image coordinates $(\mathrm{x}, \mathrm{y})$ $\mathrm{A}=$ grayscale elevation of local background $\mathrm{k}=$ factor to scale the cracks from the background $\mathrm{d}=$ distance between point $(\mathrm{x}, \mathrm{y})$ and the line passing the center of the object $\sigma=$ the spread of the profile

Therefore, a Gaussian-shaped kernel is used to match the pieces of vessels in various orientations. Such a Gaussian kernel is defined as

$$
\left.K(x, y)=-\exp \left(\frac{-x^{2}}{2 \sigma^{2}}\right)\right] \quad \text { for }|y| \leq \frac{L}{2}
$$

where $\quad K(x, y)=$ the kernel function

$\mathrm{x}=$ alignment along the direction perpendicular to the segment

$\mathrm{y}=$ alignment along the direction of the segment

$\mathrm{L}=$ the length of the segment

The kernel is rotated to match vessel segments accordingly. So the relationship between a point in a rotated orientation and in the horizontal kernel is defined as

$$
p_{i}=p\left[\begin{array}{cc}
\cos \theta_{i} & -\sin \theta_{i} \\
\sin \theta_{i} & \cos \theta_{i}
\end{array}\right]^{T}
$$

where $\quad p_{i}=$ point in the $i$ th kernel at angle $\theta_{\mathrm{i}}$

$p=$ same point in the horizontal kernel

The mean value of the filter has to be normalized to 0 in order to remove the background. Since $99 \%$ of the area under the Gaussian function lies within $\pm 3 \sigma$, the Gaussian kernel is truncated to within $3 \sigma$. Since the crack features in the present study appears brighter than the surroundings, the negative sign in the matched filter is removed to detect brighter objects rather than the initial design which aims to detect darker-than-neighbours objects.
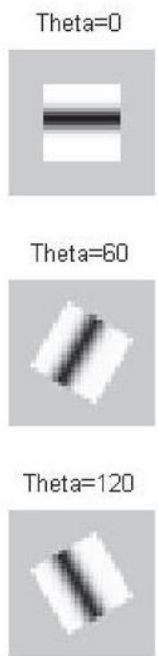

Figure 7. Illustration of the defined kernels (Adapted from Zhang et al., 2013)

The parameter $(\sigma)$ was defined here as 0.5 and the size of the kernel (L) was defined as 5 . These parameters were defined according to the common crack width and the image resolution. The rotation step is fixed to $15^{\circ}$ so that the kernel will rotate 12 times (see Figure 7) and generate 12 convolution results. At each grid, the final grid value is assigned by the maximum convolution result among the 12 results. Last an adaptive thresholding method is applied to remove noise and convert the potential crack candidates into binary image.

In the last step, a thorough noise removing process is conducted based on user-defined thresholds for both the length of connected pixels and the total area of a crack segment in order to further filter unwanted background noise and retain the true cracks.

\section{RESULTS AND DISCUSSION}

The proposed methodology is tested to extract four types of pavement cracks (longitudinal, transverse, random, and alligator cracks) with various sizes of data input. The results are shown in Figures 8 and 9. To evaluation the proposed method, the ground truth data (see Figure 8(b)) was created through a manual delineation process. The significant advantage of the proposed method is to detect cracks from the whole MLS dataset at one time. The matched filtering took only a few seconds $(<10 \mathrm{sec}$. $)$ to convolute a $2500 \times 760$ image. It was computationally efficient and simple comparing to most of state-of-the-art edge detection methods (e.g., tensor voting). And it is less affected by the noise comparing to traditional edge detection methods (e.g., Canny or Sobel edge detection). 


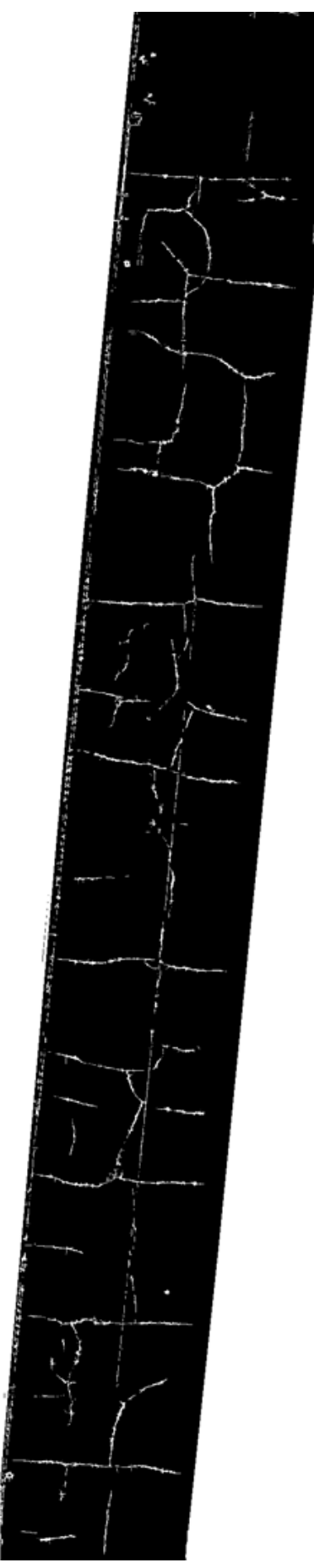

(a)

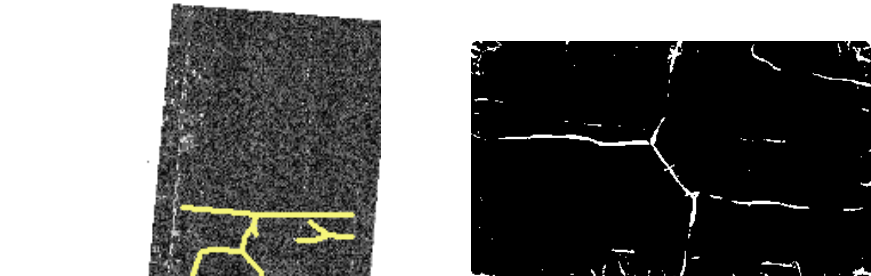

(a)

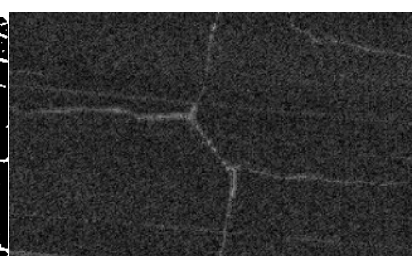

(b)

Figure 9. Results obtained by the proposed method (a) detected cracks containing a small road segment, (b) the corresponding DTM high-pass convolution result.

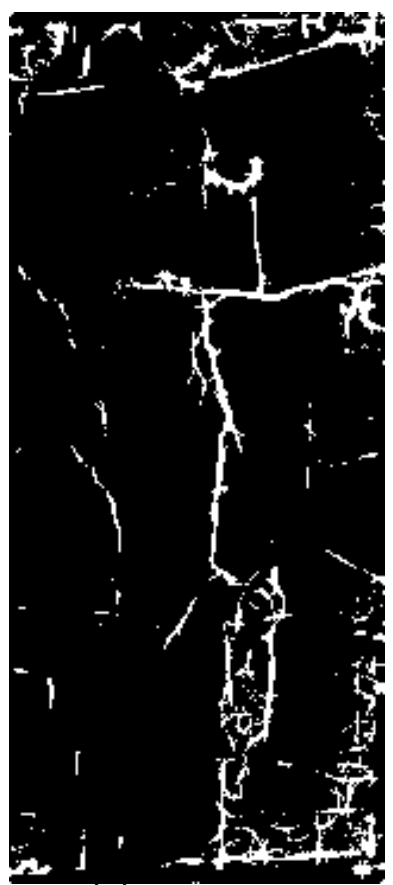

(a)

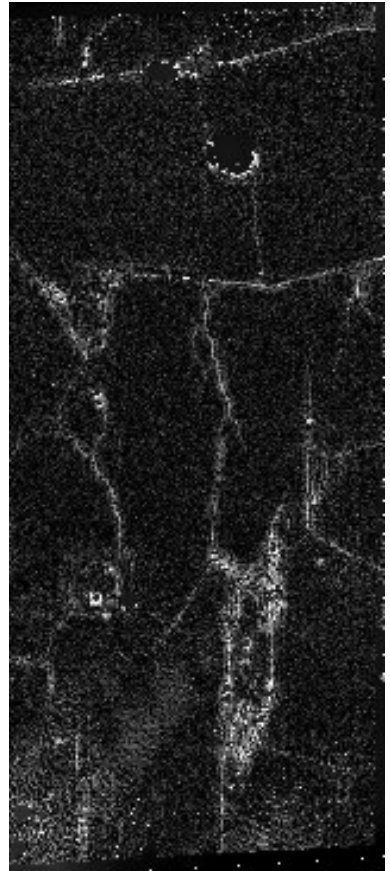

(b)

Figure 10. Results of a sample of alligator cracks: (a) detected results containing the alligator cracks, (b) the corresponding DTM high-pass convolution result.

some cracks are only a few millimetres wide by checking in the field. This is because even though each cell is $4 \mathrm{~cm}$ in size, both longitudinal and transverse cracks tend to have length in metres. For a $1 \mathrm{~m}$-long crack, it will have 25 pixels. Though MLS laser pulses may not hit every crack elements, however, if it hits at least half of them, this crack is highly likely to be detected by the proposed method.

\section{CONCLUING REMARKS}

This paper has presented a method to detect pavement cracks from MLS point clouds. The method takes the advantage of the nature of the point cloud (the 3D information) and image processing technology. Using a generic MLS system to detect pavement distress may not be the first choice for most transportation agencies due to its resolution constraint and also the computational complexity in processing 3D points. However, our study demonstrated that the MLS 3D points may also embed cracking at a sub-centimetre level. The Ministry of Transportation Ontario (MTO) classified five levels of severity of pavement cracking: very slight $(3 \mathrm{~mm})$, slight $(3 \mathrm{~mm}-13$ $\mathrm{mm})$, moderate $(13 \mathrm{~mm}-19 \mathrm{~mm})$, severe $(19 \mathrm{~mm}-25 \mathrm{~mm})$, and very severe $(>25 \mathrm{~mm}$ ) (Chong et al., 1995). Our study has demonstrated that use of VMX-450 point clouds can successfully detect cracks with up to moderate severity level. 
Cracks with very slight and slight severity remain unclear in the MLS point cloud.

The intensity of MLS point clouds have already been applied in the applications of pavement feature detection (such as road marking, manhole, and cracks). The major difference between the present study and others is the use of elevation jumps that are embedded in the 3D points rather than the intensity. This study also reveals that the $3 \mathrm{D}$ information could facilitate a series of application in relation to pavement inspection even though the data has been transformed from 3D to 2D. Besides pavement distresses, the pavement roughness would be an important factor that affects transportation safety. The generic MLS system could potentially be the equipment that can achieve all these purposes and replace the traditional surveys.

\section{REFERENCES}

Chaudhuri, S., Chatterjee, S., Katz, N., Nelson, M., Goldbaum, M., 1989. Detection of blood vessels in retinal images using two-dimensional matched filters. IEEE Transactions on Medical Imaging, 8(3), pp.263-269.

Chong, G.J., Phang, W.A., Wrong, G.A., 1995. "Manual for Condition Rating of Flexible Pavements". Distress Manifestations, Research and Development Branch, Ministry of Transportation Ontario, Toronto, Canada. http://www.ogra.org/files/REDUCED $\% 20$ FILE $\% 20$ SP024\%20Manual $\% 20$ for $\% 20 \mathrm{C}$ ondition $\% 20$ Rating $\% 20$ of $\% 20$ Flexible $\% 20$ Pavements $\% 20(1)$.p df (12 March 2016)

Guan, H., Li, J., Yu, Y., Wang, C., Chapman, M.A., Yang, B., 2014. Using mobile laser scanning data for automated extraction of road markings. ISPRS Journal of Photogrammetry and Remote Sensing, 87, pp. 93-107

Guan, H., Li, J., Yu, Y., Wang, C., Chapman, M., Yang, B., 2014. Using mobile laser scanning data for automated extraction of road markings. ISPRS Journal of Photogrammetry and Remote Sensing, 87, pp. 93-107.

Guan, H., Li, J., Yu, Y., Ji, Z., Wang, C., 2015. Using mobile LiDAR data for rapidly updating road markings. IEEE Transactions on Intelligent Transportation Systems, 16(5), pp.2457-2466.

Guan, H., Li, J., Yu, Y., Chapman, M.A., Wang, H., Wang, C., Zhai, R., 2015. Iterative tensor voting for pavement crack extraction using mobile laser scanning data. IEEE Transactions on Geoscience and Remote Sensing, 53(3), pp.1527-1537.

Smith, T., Tighe, S., Fung, R., 2001. Paper presented at the 2001 Annual Conference of the Transportation Association of Canada, Halifax, Nova Scotia http://www.cement.ca/images/stories/concrete pavements in canada -usage and performance. $\operatorname{pdf}(12$ March 2016).

Tsai, Y.C.J., Li, F., 2012. Critical assessment of detecting asphalt pavement cracks under different lighting and low intensity contrast conditions using emerging $3 \mathrm{D}$ laser technology. Journal of Transportation Engineering, 138(5), pp. 649-656.

Wang, H., Luo, H., Wen, C., Cheng, J., Chen, Y., Wang, C., Li, J., 2015. Road boundaries detection based on local normal saliency from mobile laser scanning data. IEEE Geoscience and Remote Sensing Letters, 12(10), pp.2085-2089.
Wen, C., Li, J., Luo, H., Yu, Y., Cai, Z., Wang, H., Wang, C., 2016. Spatial-related traffic sign inspection for inventory purposes using mobile laser scanning data. IEEE Transactions on Intelligent Transportation Systems, 17(1), pp. 27-37.

Wu, F., Wen, C., Guo, Y., Wang, J., Yu, Y., Chen, Y., Wang, C., Li, J., 2016. Rapid localization and extraction of street light poles in mobile LiDAR point clouds: a supervoxels-based approach, IEEE Transactions on Intelligent Transportation Systems, under revision.

Xu, B., Huang, Y., 2003. Development of an Automatic Pavement Surface Distress Inspection System, Center for Transportation Research, The University of Texas at Austin, Report No. FHWA/TX-05/7-4975-1.

Yang, B., Fang, L., Li, Q., Li, J., 2012. Automated extraction of road markings from mobile lidar point cloud. Photogrammetric Engineering \& Remote Sensing, 78(4), pp.331-338.

Yu, Y., Li, J., Guan, H., Wang, C., 2014. Automated detection of road manhole and sewer well covers from mobile LiDAR point clouds. IEEE Geoscience and Remote Sensing Letters, 11(9), pp.1549-1553.

Yu, Y., Guan, H., Ji, Z., 2015a. Automated detection of urban road manhole covers using mobile laser scanning data. IEEE Transactions on Intelligent Transportation Systems, 16(6), pp. 3258-3269.

Yu, Y., Li, J., Guan, H., Wang, C., Yu, J., 2015b. Semi-automated extraction of street light poles from mobile LiDAR point-clouds. IEEE Transactions on Geoscience and Remote Sensing, 53(3), pp.1374 -1386.

Yu, Y., Li, J., Guan, H., Jia, F., Wang, C., 2015c. Learning hierarchical features for automated extraction of road markings from 3-D mobile LiDAR point clouds. IEEE Journal of Selected Topics in Applied Earth Observations and Remote Sensing, 8(2), pp.709-726.

Yu, Y., Li, J., Guan, H., Wang, C., Wen, C., 2016a. Bag-of-contextual-visual-words for road scene object detection from mobile laser scanning data. IEEE Transactions on Intelligent Transportation Systems, 13(4), pp.520-524.

Yu, Y., Li, J., Wen, C., Guan, H., Luo, H., Wang, C., 2016 b. Bag-of-visual-phrases and hierarchical deep models for traffic sign detection and recognition in mobile laser scanning data. ISPRS Journal of Photogrammetry and Remote Sensing, 113, pp.106-123.

Yu, Y., Li, J., Guan, H., Wang, C., 2016c. Automated detection of three-dimensional cars in mobile laser scanning point clouds using DBM-Hough-Forests. IEEE Transactions on Geoscience and Remote Sensing, 99, pp.1-13..

Zhang, A., Li, Q., Wang, K., Qiu, S., 2013. Matched filtering algorithm for pavement cracking detection. Journal of the Transportation Research Board, 2367, pp. 30-42. 